\title{
A fuzzy characterization of QF rings
}

\author{
Wenxi Li ${ }^{1}$, Jianlong Chen ${ }^{2}$ and Liang Shen ${ }^{2 *}$
}

${ }^{\text {*Correspondence: }}$ Ishen@seu.edu.cn

${ }^{2}$ Department of Mathematics, Southeast University, Nanjing, 210096, China

Full list of author information is available at the end of the article

\begin{abstract}
Let $R$ be a ring. $R$ is called a quasi-Frobenius (QF) ring if $R$ is right artinian and $R_{R}$ is an injective right $R$-module. In this article, we introduce (weak) fuzzy homomorphisms of modules to obtain a fuzzy characterization of QF rings. We also obtain some fuzzy characterizations of right artinian rings and right CF rings. These results throw new light on the research of $\mathrm{QF}$ rings and the related $\mathrm{CF}$ conjecture.
\end{abstract}

MSC: 03E72; 16L60

Keywords: fuzzy homomorphisms; weak fuzzy homomorphisms; artinian rings; QF rings

\section{Introduction}

Recall that a fuzzy subset of a nonempty set $X$ is a map $f$ from $X$ into the closed interval $[0,1]$. The notion of fuzzy subset of a set was firstly introduced by Zadeh [1]. Then this important ideal has been applied to various algebraic structures such as groups and rings and so on (see [2-9] etc.). In this article, we introduce some special fuzzy subsets of modules to characterize quasi-Frobenius (QF) rings.

QF rings were introduced by Nakayama [10] as generalizations of group algebras of a finite group over a field. A ring $R$ is called quasi-Frobenius $(Q F)$ if the right $R$-module $R_{R}$ is both artinian and injective. QF rings became an important algebraic structure because of their beautiful characterizations and nice applications (see [11-16] etc.). For example, a ring $R$ is QF if and only if every right $R$-module can be embedded into a free right $R$-module. Many results of QF rings have been applied into coding theory. During the progress of research on QF rings, many important conjectures arose. One of them is the $C F$ conjecture (see $[17,18]$ etc.). It says that every right $C F$ ring is right artinian. Recall that a ring $R$ is called right $C F$ if every cyclic right $R$-module can be embedded into a free right $R$-module.

Firstly, we introduce the fuzzy homomorphism and weak fuzzy homomorphism of $R$ modules in Section 2. Then in Section 3, we use weak fuzzy homomorphisms to give a characterization of injective right $R$-modules. We also obtain some new fuzzy characterizations of right artinian rings. In Section 4, we give a fuzzy characterization of right CF rings. We also give an approach to the CF conjecture through fuzzy viewpoints. Then based on the results we have obtained, we finally get a fuzzy characterization of QF rings.

\section{Definitions and examples}

Throughout the paper, $R$ is an associative ring with identity and all modules are unitary. For a subset $X$ of a ring $R$, the right annihilator of $X$ in $R$ is $\mathbf{r}(X)=\{r \in R: x r=0$ for all $x \in$

○2014 Li et al.; licensee Springer. This is an Open Access article distributed under the terms of the Creative Commons Attribution License (http://creativecommons.org/licenses/by/2.0), which permits unrestricted use, distribution, and reproduction in any medium, provided the original work is properly cited. 
$X$. We write $M_{R}$ to indicate that $M$ is a right $R$-module. Let $M_{R}$ and $N_{R}$ be two right $R$-modules. $\operatorname{Hom}_{R}(M, N)$ denotes the set of all right $R$-module homomorphisms from $M_{R}$ to $N_{R} . A \times B$ means the Cartesian cross product of two sets $A$ and $B$. We use $\operatorname{Im}(f)$ to denote the image of a map $f$. For much more notations one is referred to [19].

We now consider the following conditions of a fuzzy subset $f$ of $M_{R} \times N_{R}$.

(1) $\forall x \in M, \exists y \in N$ such that $f(x, y)>0$;

(1') $\exists x \in M, \exists y \in N$ such that $f(x, y)>0$;

(2) $\forall x \in M, \forall y_{1}, y_{2} \in N, f\left(x, y_{1}\right)>0$ and $f\left(x, y_{2}\right)>0$ implies $y_{1}=y_{2}$;

(3) $\forall x_{1}, x_{2} \in M, \forall y \in N$,

$$
f\left(x_{1}+x_{2}, y\right) \geq \sup \left\{\min \left\{f\left(x_{1}, y_{1}\right), f\left(x_{2}, y_{2}\right)\right\}: y_{1}+y_{2}=y, y_{1}, y_{2} \in N\right\}
$$

(4) $\forall x \in M, \forall y \in N, \forall r \in R, f(x r, y) \geq \sup \left\{f\left(x, y_{1}\right): y=y_{1} r, y_{1} \in N\right\}$.

Definition 2.1 If $f$ satisfies (1), (2), (3), and (4) of the above conditions, $f$ is called a fuzzy homomorphism from $M_{R}$ to $N_{R}$. If $f$ satisfies $\left(1^{\prime}\right),(2),(3)$, and (4) of the above conditions, $f$ is called a weak fuzzy homomorphism from $M_{R}$ to $N_{R}$. We will use $\operatorname{FHom}_{R}(M, N)$ (resp., WFHom $_{R}(M, N)$ ) to denote the set of all fuzzy homomorphisms (resp., weak fuzzy homomorphisms) from $M_{R}$ to $N_{R}$. It is clear that $\operatorname{FHom}_{R}(M, N) \subseteq \mathrm{WFHom}_{R}(M, N)$.

Example 2.2 Let $u \in \operatorname{Hom}_{R}(M, N) \cdot f_{u}$ is a fuzzy subset of $M \times N$ constructed by

$$
f_{u}(x, y)= \begin{cases}1, & x \in M, \text { and } y=u(x) \\ 0, & \text { others. }\end{cases}
$$

Then $f_{u} \in \operatorname{FHom}_{R}(M, N)$.

Proof The conditions (1) and (2) of $f_{u}$ are satisfied obviously. Let $x_{1}, x_{2} \in M$ and $y \in N$. If $y=u\left(x_{1}+x_{2}\right)$, it is clear that

$$
1=f_{u}\left(x_{1}+x_{2}, y\right) \geq \sup \left\{\min \left\{f_{u}\left(x_{1}, y_{1}\right), f_{u}\left(x_{2}, y_{2}\right)\right\}: y_{1}+y_{2}=y, y_{1}, y_{2} \in N\right\} .
$$

If $y \neq u\left(x_{1}+x_{2}\right)$ and $y_{1}+y_{2}=y$, then either $u\left(x_{1}\right) \neq y_{1}$ or $u\left(x_{2}\right) \neq y_{2}$. Thus, $\min \left\{f_{u}\left(x_{1}, y_{1}\right)\right.$, $\left.f_{u}\left(x_{2}, y_{2}\right)\right\}=0$. So

$$
0=f_{u}\left(x_{1}+x_{2}, y\right) \geq 0=\sup \left\{\min \left\{f_{u}\left(x_{1}, y_{1}\right), f_{u}\left(x_{2}, y_{2}\right)\right\}: y_{1}+y_{2}=y\right\} .
$$

Hence $f_{u}$ satisfies the condition (3). For the condition (4), let $x \in M, y \in N, r \in R$. If $y=$ $u(x r)$, it is clear that $1=f_{u}(x r, y) \geq \sup \left\{f_{u}\left(x, y_{1}\right): y=y_{1} r, y_{1} \in N\right\}$. If $y \neq u(x r)$ and $y=y_{1} r$, then $y_{1} \neq u(x)$. So $f_{u}\left(x, y_{1}\right)=0$. Hence $0=f_{u}(x r, y) \geq 0=\sup \left\{f_{u}\left(x, y_{1}\right): y=y_{1} r, y_{1} \in N\right\}$. Thus, $f_{u} \in \operatorname{FHom}_{R}(M, N)$.

Remark 2.3 Let $f \in \mathrm{WFHom}_{R}(M, N)$.

(i) According to the condition (3), $\forall x_{1}, x_{2} \in M, \forall y_{1}, y_{2} \in N$, $f\left(x_{1}+x_{2}, y_{1}+y_{2}\right) \geq \min \left\{f\left(x_{1}, y_{1}\right), f\left(x_{2}, y_{2}\right)\right\}$. According to the conditions (3) and (4), $\forall x \in M, \forall y \in N, f(0,0) \geq \min \{f(x, y), f(-x,-y)\}=f(x, y)=f(-x,-y)$. 
(ii) Set $t \in(0,1]$ and $M_{t}=\left\{x \in M_{R}: \exists y \in N, f(x, y) \geq t\right\}$. If $M_{t}$ is not empty, according to the conditions (3) and (4), $M_{t}$ is a right $R$-submodule of $M_{R}$.

(iii) Let $K_{R}$ be a submodule of $M_{R}$ such that $\left.f\right|_{K \times N} \in \operatorname{FHom}_{R}(K, N)$. Then by the conditions (1) and (2), for each $x \in K$, there exists a unique $y_{x} \in N$ such that $f\left(x, y_{x}\right)>0$. Now define a map $u: K \rightarrow N$ with $u(x)=y_{x}$. Again by the conditions (2), (3), and (4), it is not difficult to see that $u \in \operatorname{Hom}_{R}(K, N)$.

Definition 2.4 Let $f_{1}, f_{2} \in \operatorname{WFHom}_{R}(M, N)$, we say $f_{1} \leq f_{2}$ if $f_{1}(x, y) \leq f_{2}(x, y)$ for all $x \in M$, $y \in N$. $f_{1} \wedge f_{2}$ is defined by $\left(f_{1} \wedge f_{2}\right)(x, y)=\min \left\{f_{1}(x, y), f_{2}(x, y)\right\}, \forall x \in M, y \in N$. It is easy to prove that if $f_{1}, f_{2} \in \operatorname{WFHom}_{R}(M, N)$, then $f_{1} \wedge f_{2} \in \operatorname{WFHom}_{R}(M, N)$. It is also clear that $f_{1} \wedge f_{2} \leq f_{1}$ and $f_{1} \wedge f_{2} \leq f_{2}$.

Definition 2.5 A weak fuzzy homomorphism $f \in \operatorname{WFHom}_{R}(M, N)$ is said to be extendable if there exists $g \in \operatorname{FHom}_{R}(M, N)$ such that $f \leq g$.

Example 2.6 Let $M_{R}$ be a non-artinian right $R$-module. Then $M$ has an infinite descending chain of submodules $M=M_{1} \supsetneq M_{2} \supsetneq M_{3} \cdots$. Set $N=\bigcap_{i} M_{i}$. Now we define a fuzzy subset $f$ of $M \times M$ by

$$
f(x, y)= \begin{cases}1, & x=y \in N, \\ 1-\frac{1}{n+1}, & x=y \in M_{n}, x \notin M_{n+1}, \\ 0, & \text { others. }\end{cases}
$$

Then $f \in \operatorname{FHom}_{R}(M, M)$.

Proof It is obvious that $f$ satisfies the conditions (1) and (2).

For the condition (3), let $x_{1}, x_{2}, y \in R$ with $y=y_{1}+y_{2}$, where $y_{1}, y_{2} \in R$. We only need to consider the following three cases.

Case 1: $\min \left\{f\left(x_{1}, y_{1}\right), f\left(x_{2}, y_{2}\right)\right\}=0$. It is clear that $f\left(x_{1}+x_{2}, y\right) \geq \min \left\{f\left(x_{1}, y_{1}\right), f\left(x_{2}, y_{2}\right)\right\}$.

Case 2: $0<\min \left\{f\left(x_{1}, y_{1}\right), f\left(x_{2}, y_{2}\right)\right\}<1$. We can suppose that $f\left(x_{1}, y_{1}\right)=1-\frac{1}{j+1}$ or 1 , $f\left(x_{2}, y_{2}\right)=1-\frac{1}{k+1}$ and $j \geq k$. Then $I \subseteq I_{j} \subseteq I_{k}$ and $x_{1}=y_{1} \in M_{j}$ or $N, x_{2}=y_{2} \in I_{k}$. So $x_{1}+x_{2}=y_{1}+y_{2} \in I_{k}$. Hence $f\left(x_{1}+x_{2}, y\right) \geq 1-\frac{1}{k+1}=\min \left\{f\left(x_{1}, y_{1}\right), f\left(x_{2}, y_{2}\right)\right\}$.

Case 3: $\min \left\{f\left(x_{1}, y_{1}\right), f\left(x_{2}, y_{2}\right)\right\}=1$. Then $x_{1}=y_{1} \in N$ and $x_{2}=y_{2} \in N$. So $f\left(x_{1}+x_{2}, y\right)=$ $1 \geq \min \left\{f\left(x_{1}, y_{1}\right), f\left(x_{2}, y_{2}\right)\right\}$.

From the above three cases, it is clear that $f$ satisfies the condition (3).

Finally, let $x, r, y \in R$ with $y=y_{1} r$, where $y_{1} \in R$. If $f\left(x, y_{1}\right)=0$, then $f(x r, y) \geq f\left(x, y_{1}\right)$. If $f\left(x, y_{1}\right)=1-\frac{1}{k+1}$ for a positive integer $k$, then $x=y_{1} \in M_{k}$. So $x r=y_{1} r \in M_{k}$. Hence $f(x r, y) \geq 1-\frac{1}{k+1}=f\left(x, y_{1}\right)$. If $f\left(x, y_{1}\right)=1$, then $x=y_{1} \in N$. So $x r=y \in N$. Thus $f(x r, y) \geq$ $f\left(x, y_{1}\right)$. Therefore, $f(x r, y) \geq \sup \left\{f\left(x, y_{1}\right): y=y_{1} r, y_{1} \in R\right\}$ for all $x \in R, y \in R$ and $r \in R$. Then $f$ satisfies the condition (4).

Definition 2.7 A weak fuzzy homomorphism $f \in \operatorname{WFHom}_{R}(M, N)$ is said to be bounded if there exists $t \in(0,1]$ such that, $\forall m \in M$ and $\forall n \in N, f(m, n) \geq t$ or $f(m, n)=0$.

Example 2.8 Let $M_{R}$ be a non-noetherian right $R$-module. Then $M$ has an infinite ascending chain of submodules $M_{1} \subsetneq M_{2} \subsetneq M_{3} \ldots$. Now we define a fuzzy subset $f$ of $M \times M$ 
by

$$
f(x, y)= \begin{cases}1, & x \in M_{1}, y=0 \\ \frac{1}{k}, & x \in M_{k}, x \notin M_{k-1}, k \geq 2, y=0, \\ 0, & \text { others. }\end{cases}
$$

Then $f \in \mathrm{WFHom}_{R}(M, M)$. In particular, $f$ is extendable and not bounded.

Proof By a similar discussion as that in Example 2.6, we have $f \in \operatorname{WFHom}_{R}(M, M)$. It is obvious that $f$ is not bounded. Now set

$$
g(x, y)= \begin{cases}1, & x \in M, y=0 \\ 0, & \text { others }\end{cases}
$$

It is clear that $g \in \operatorname{FHom}_{R}(M, M)$ and $f \leq g$. So $f$ is extendable.

\section{Fuzzy characterizations of injective modules and artinian rings}

According to Baer's Criterion, a right $R$-module $M_{R}$ is said to be injective if every homomorphism from a right ideal $I$ of $R$ to $M_{R}$ can be extended to a homomorphism from $R_{R}$ to $M_{R}$.

Theorem 3.1 Let $R$ be a ring and $M_{R}$ a right $R$-module. Then $M$ is injective if and only if every $f \in \mathrm{WFHom}_{R}(R, M)$ is extendable.

Proof $(\Leftarrow)$ Let $I$ be a right ideal of $R$. Suppose $u \in \operatorname{Hom}_{R}(I, M)$, we will show that $u$ can be extended to a homomorphism $v \in \operatorname{Hom}_{R}(R, M)$. Firstly we construct a fuzzy subset of $R \times M$ by

$$
f(x, y)= \begin{cases}1, & x \in I, \text { and } y=u(x) \\ 0, & \text { others }\end{cases}
$$

By a similar proof of Example 2.2, $f \in \mathrm{WFHom}_{R}(R, M)$. Since $f$ is extendable, there exists some $g \in \operatorname{FHom}_{R}(R, M)$ such that $f \leq g$. Now define $v: R \rightarrow M$ via $v(x)=y$, where $g(x, y)>0$. According to Remark 2.3(iii), $v \in \operatorname{Hom}_{R}(R, M)$. It is easy to see that $\left.v\right|_{I}=u$. This shows that $M$ is an injective right $R$-module.

$(\Rightarrow)$ Assume that $M$ is an injective right $R$-module and $f \in \operatorname{WFHom}_{R}(R, M)$. Set $I=$ $\sum_{0 \neq t \in \operatorname{Im} f} R_{t}$. By Remark 2.3(ii), $I$ is a right ideal of $R$. Now define $u: I \rightarrow M$ via $u(x)=y$, where $f(x, y)>0$. According to Remark 2.3(iii), $u \in \operatorname{Hom}_{R}(I, M)$. Since $M$ is injective as a right $R$-module, $u$ can be extended to a homomorphism $v$ from $R_{R}$ to $M_{R}$. By Example 2.2, we have $f_{v} \in \operatorname{FHom}_{R}(R, M)$. It is clear that $f \leq f_{v}$. So $f$ is extendable.

Next we will give some new fuzzy characterizations of right artinian rings. Recall that a fuzzy subset $\mu$ of a ring $R$ is called a fuzzy left (right) ideal of $R$ if $\mu$ satisfies: (i) $\mu(x-y) \geq$ $\min \{\mu(x), \mu(y)\}$; (ii) $\mu(x y) \geq \mu(y)(\mu(x y) \geq \mu(x))$ for all $x, y \in R$. A fuzzy subset $f$ is called finite valued if $\operatorname{Im} f$ is a finite set. If $\operatorname{Im} f$ is an infinite set, $f$ is called infinite valued.

Theorem 3.2 The following conditions are equivalent for a ring $R$. 
(a) $R$ is right artinian.

(b) Every fuzzy right ideal of $R$ is finite valued.

(c) For every $f \in \mathrm{WFHom}_{R}\left(R_{R}, R_{R}\right), f$ is finite valued.

(d) For every $f \in \mathrm{FHom}_{R}\left(R_{R}, R_{R}\right)$, $f$ is finite valued.

Proof (a) $\Leftrightarrow$ (b) See [6, Theorem 3.2].

(b) $\Rightarrow$ (c) Suppose $f \in$ WFHom $_{R}\left(R_{R}, R_{R}\right)$. We can define a fuzzy subset $\mu$ of $R$ by

$$
\mu(x)= \begin{cases}t, & f(x, y)=t>0 \text { for some } y \in R \\ 0, & \text { others. }\end{cases}
$$

Since $f \in \operatorname{WFHom}_{R}\left(R_{R}, R_{R}\right)$, according to the condition (4), $f(x r, y r) \geq f(x, y), \forall x, y, r \in R$. We have $\mu(x r) \geq \mu(x)$. For any $x_{1}, x_{2} \in R$, if $\mu\left(x_{1}\right)=0$ or $\mu\left(x_{2}\right)=0$, it is clear that $\mu\left(x_{1}-\right.$ $\left.x_{2}\right) \geq \min \left\{\mu\left(x_{1}\right), \mu\left(x_{2}\right)\right\}$. If $\mu\left(x_{1}\right)>0$ and $\mu\left(x_{2}\right)>0$, then there exist $y_{1}, y_{2} \in R$ such that $\mu\left(x_{1}\right)=f\left(x_{1}, y_{1}\right)$ and $\mu\left(x_{2}\right)=f\left(x_{2}, y_{2}\right)$. Again since $f \in \mathrm{WFHom}_{R}\left(R_{R}, R_{R}\right)$, according to the condition (3) and Remark 2.3(i), we have

$$
f\left(x_{1}-x_{2}, y_{1}-y_{2}\right) \geq \min \left\{f\left(x_{1}, y_{1}\right), f\left(-x_{2},-y_{2}\right)\right\}=\min \left\{f\left(x_{1}, y_{1}\right), f\left(x_{2}, y_{2}\right)\right\} .
$$

Therefore $\mu\left(x_{1}-x_{2}\right) \geq \min \left\{\mu\left(x_{1}\right), \mu\left(x_{2}\right)\right\}$. So $\mu$ is a fuzzy right ideal of $R$. Since $\operatorname{Im} f \subseteq$ $\operatorname{Im} \mu \cup\{0\}$ and $\mu$ is finite valued, $f$ is finite valued.

(c) $\Rightarrow$ (d) is obvious.

(d) $\Rightarrow$ (a) Assume that $R$ is not right artinian. Let $R=I_{1} \supsetneq I_{2} \supsetneq I_{3} \cdots$ be a descending chain of right ideals of $R$ and $I=\bigcap_{i} I_{i}$. Define a fuzzy subset $f$ of $R \times R$ by

$$
f(x, y)= \begin{cases}1, & x=y \in I, \\ 1-\frac{1}{n+1}, & x=y \in I_{n}, x \notin I_{n+1}, \\ 0, & \text { others. }\end{cases}
$$

By Example 2.6, $f \in \mathrm{FHom}_{R}\left(R_{R}, R_{R}\right)$. But $f$ is infinite valued. This is a contradiction. So $R$ is right artinian.

\section{Fuzzy characterizations of right CF rings and QF rings}

In this section, we will firstly give a fuzzy characterization of right $C F$ ring. It is well known that a ring $R$ is right $C F$ if and only if for every right ideal $I$ of $R$, there exist $x_{1}, \ldots, x_{n} \in R$ such that $I=\mathbf{r}\left(x_{1}, \ldots, x_{n}\right)$ (see [14, Lemma 7.2]).

Theorem 4.1 $A$ ring $R$ is a right $C F$ ring if and only if for every bounded and extendable $f \in \operatorname{WFHom}\left(R_{R}, R_{R}\right)$, there exist $F_{1}, \ldots, F_{n} \in \operatorname{FHom}_{R}\left(R_{R}, R_{R}\right)$ such that $f=F_{1} \wedge F_{2} \wedge \cdots \wedge F_{n}$.

Proof $(\Rightarrow)$ Suppose $R$ is a right CF ring and $f \in \operatorname{WFHom}(R, R)$ is bounded and extendable. Then there exist $F \in \operatorname{FHom}_{R}\left(R_{R}, R_{R}\right)$ and $s \in(0,1]$ such that $f \leq F$ and $f(x, y) \geq s$ for all $x, y \in R$ with $f(x, y)>0$. Set $I=\sum_{0 \neq t \in \operatorname{Im} f} R_{t}$. By Remark 2.3(ii), $I$ is a right ideal of $R$. Since $R$ is a right CF ring, there exist $c_{1}, \ldots, c_{n} \in R$ such that $I=\mathbf{r}\left(c_{1}, \ldots, c_{n}\right)$. To be convenient, we assume that $c_{1}=0$. Since $F \in \operatorname{FHom}_{R}\left(R_{R}, R_{R}\right)$, for the identity $1 \in R$, there exists $c \in R$ 
such that $F(1, c)>0$. We now can construct fuzzy subsets $F_{1}, F_{2}, \ldots, F_{n}$ of $R \times R$ by

$$
F_{i}(x, y)= \begin{cases}f(x, y), & x \in I, y=c x \\ s, & x \notin I, y=\left(c-c_{i}\right) x, \quad i=1,2, \ldots, n \\ 0, & \text { others. }\end{cases}
$$

At first, we show that $F_{i} \in \mathrm{FHom}_{R}\left(R_{R}, R_{R}\right), i=1,2, \ldots, n$.

(a) If $x \in I$, according to Remark 2.3(i), there exists $y \in R$, such that $f(x, y)>0$. As $F \in$ $\mathrm{FHom}_{R}\left(R_{R}, R_{R}\right)$, by the condition (4), $F(x, c x) \geq F(1, c)>0$. Then by the condition (2), we have $F(x, y)=0$ for all $y \neq c x$. Since $f \leq F, f(x, y)=0$ for all $y \neq c x$. This implies that $f(x, c x)=$ $f(x, y)>0$. Therefore, $F_{i}(x, c x)=f(x, c x)>0$. If $x \notin I$, let $y=\left(c-c_{i}\right) x$, then $F_{i}(x, y)=s>0$. So $F_{i}$ satisfies the condition (1).

(b) If $x \in I$ and $y_{1}, y_{2} \in R$ with $F_{i}\left(x, y_{1}\right)>0$ and $F_{i}\left(x, y_{2}\right)>0$, then $y_{1}=y_{2}=c x$. If $x \notin I$ and $y_{1}, y_{2} \in R$ with $F_{i}\left(x, y_{1}\right)>0$ and $F_{i}\left(x, y_{2}\right)>0$, then $y_{1}=y_{2}=\left(c-c_{i}\right) x$. So $F_{i}$ satisfies the condition (2).

(c) Let $x_{1}, x_{2}, y \in R$ with $y=y_{1}+y_{2}$, where $y_{1}, y_{2} \in R$.

If $\min \left\{F_{i}\left(x_{1}, y_{1}\right), F_{i}\left(x_{2}, y_{2}\right)\right\}=0$, then $F_{i}\left(x_{1}+x_{2}, y\right) \geq \min \left\{F_{i}\left(x_{1}, y_{1}\right), F_{i}\left(x_{2}, y_{2}\right)\right\}$. If $\min \left\{F_{i}\left(x_{1}\right.\right.$, $\left.\left.y_{1}\right), F_{i}\left(x_{2}, y_{2}\right)\right\}>0$, we only need to discuss the following three cases.

Case 1: $x_{1}, x_{2} \in I$. Then $y_{1}=c x_{1}$ and $y_{2}=c x_{2}$.

So $y=c\left(x_{1}+x_{2}\right)$, and

$$
\begin{aligned}
F_{i}\left(x_{1}+x_{2}, y\right) & =f\left(x_{1}+x_{2}, y\right) \\
& \geq \min \left\{f\left(x_{1}, y_{1}\right), f\left(x_{2}, y_{2}\right)\right\} \\
& =\min \left\{F_{i}\left(x_{1}, y_{1}\right), F_{i}\left(x_{2}, y_{2}\right)\right\} .
\end{aligned}
$$

Case 2: $x_{1} \in I$ and $x_{2} \notin I$. Then $y_{1}=c x_{1}, y_{2}=\left(c-c_{i}\right) x_{2}$ and $x_{1}+x_{2} \notin I$.

So $\min \left\{F_{i}\left(x_{1}, y_{1}\right), F_{i}\left(x_{2}, y_{2}\right)\right\}=s$. Since $I=\mathbf{r}\left(c_{1}, \ldots, c_{n}\right), c_{i} x_{1}=0$. Then $y_{1}+y_{2}=c x_{1}+(c-$ $\left.c_{i}\right) x_{2}=\left(c-c_{i}\right)\left(x_{1}+x_{2}\right)$. Thus, $F_{i}\left(x_{1}+x_{2}, y\right)=s=\min \left\{F_{i}\left(x_{1}, y_{1}\right), F_{i}\left(x_{2}, y_{2}\right)\right\}$.

Case 3: $x_{1} \notin I$ and $x_{2} \notin I$. Then $y_{1}=\left(c-c_{i}\right) x_{1}$ and $y_{2}=\left(c-c_{i}\right) x_{2}$.

So $\min \left\{F_{i}\left(x_{1}, y_{1}\right), F_{i}\left(x_{2}, y_{2}\right)\right\}=s$ and $y_{1}+y_{2}=\left(c-c_{i}\right)\left(x_{1}+x_{2}\right)$. If $\left(x_{1}+x_{2}\right) \in I$, then $y_{1}+y_{2}=$ $\left(c-c_{i}\right)\left(x_{1}+x_{2}\right)=c\left(x_{1}+x_{2}\right)$ because $c_{i} x_{1}=c_{i} x_{2}=0$. Thus, $F_{i}\left(x_{1}+x_{2}, y\right)=f\left(x_{1}+x_{2}, y\right) \geq s=$ $\min \left\{F_{i}\left(x_{1}, y_{1}\right), F_{i}\left(x_{2}, y_{2}\right)\right\}$. If $\left(x_{1}+x_{2}\right) \notin I$, then $y_{1}+y_{2}=\left(c-c_{i}\right)\left(x_{1}+x_{2}\right)$. So $F_{i}\left(x_{1}+x_{2}, y\right)=s \geq$ $\min \left\{F_{i}\left(x_{1}, y_{1}\right), F_{i}\left(x_{2}, y_{2}\right)\right\}$.

Therefore, $F_{i}$ satisfies the condition (3).

(d) For all $x, y_{1} r \in R$ with $y=y_{1} r$, where $y_{1} \in R$. If $F_{i}\left(x, y_{1}\right)=0, F_{i}(x r, y) \geq F_{i}\left(x, y_{1}\right)$. If $F_{i}\left(x, y_{1}\right)>0$, we consider the following two cases.

Case 1: $x \in I$. Then $y_{1}=c x$ and $F_{i}\left(x, y_{1}\right)=f\left(x, y_{1}\right)$. Thus, $x r \in I$ and $y=y_{1} r=c x r$. So $F_{i}(x r, y)=f(x r, y) \geq f\left(x, y_{1}\right)=F_{i}\left(x, y_{1}\right)$.

Case 2: $x \notin I$. Then $y_{1}=\left(c-c_{i}\right) x$ and $F_{i}\left(x, y_{1}\right)=s$. Since $x r \in I, c_{i} x r=0$. Then $y=y_{1} r=$ $\left(c-c_{i}\right) x r=c x r$. Thus, $F_{i}(x r, y)=f(x r, y) \geq s=F_{i}\left(x, y_{1}\right)$. If $x r \notin I$ then $y=y_{1} r=\left(c-c_{i}\right) x r$. So $F_{i}(x r, y)=s \geq F_{i}\left(x, y_{1}\right)$.

Hence $F_{i}$ satisfies the condition (4).

From the above, $F_{i} \in \mathrm{FHom}_{R}\left(R_{R}, R_{R}\right)$ for $i=1,2, \ldots, n$.

Next we show that $f=F_{1} \wedge F_{2} \wedge \cdots \wedge F_{n}$. 
Case 1: $x \in I$. If $y=c x$, then $F_{i}(x, y)=f(x, y), i=1,2, \ldots, n$. So $f(x, y)=\min \left\{F_{1}(x, y), F_{2}(x, y)\right.$, $\left.\ldots, F_{n}(x, y)\right\}$. By (a) in the above proof, $f(x, c x)>0$. If $y \neq c x$, then $f(x, y)=0$. So $F_{i}(x, y)=0$, $i=1, \ldots, n$. Hence $f(x, y)=\min \left\{F_{1}(x, y), F_{2}(x, y), \ldots, F_{n}(x, y)\right\}$ for every $x \in I$.

Case 2: $x \notin I$. By the definition of $I, \forall y \in R, f(x, y)=0$. If $y \neq\left(c-c_{j}\right) x$ for some $j \in$ $\{1,2, \ldots, n\}$, then $F_{j}(x, y)=0$. Thus, $f(x, y)=\min \left\{F_{1}(x, y), F_{2}(x, y), \ldots, F_{n}(x, y)\right\}$. If $y=\left(c-c_{i}\right) x$ for any $i \in\{1,2, \ldots, n\}$, since $c_{1}=0, c x=\left(c-c_{1}\right) x=\left(c-c_{2}\right) x=\cdots=\left(c-c_{n}\right) x$. So $c_{i} x=0$, $i=1,2, \ldots, n$. Hence $x \in \mathbf{r}\left(c_{1}, \ldots, c_{n}\right)=I$. This is a contradiction.

So $f=F_{1} \wedge F_{2} \wedge \cdots \wedge F_{n}$.

$(\Leftarrow)$ Let $I$ be a right ideal of $R$. We can construct a fuzzy subset $f$ of $R \times R$ by

$$
f(x, y)= \begin{cases}1, & x \in I, \text { and } y=0 \\ 0, & \text { others. }\end{cases}
$$

It is easy to see that $f \in \mathrm{WFHom}_{R}\left(R_{R}, R_{R}\right)$ and it is bounded and extendable. So there exist $F_{1}, \ldots, F_{n} \in \mathrm{FHom}_{R}\left(R_{R}, R_{R}\right)$ such that $f=F_{1} \wedge F_{2} \wedge \cdots \wedge F_{n}$. Then there exists $c_{i} \in R$ such that $F_{i}\left(1, c_{i}\right)>0, i=1, \ldots, n$. By the condition (4), for each $x \in R, F_{i}\left(x, c_{i} x\right) \geq F_{i}\left(1, c_{i}\right)>0$, $i=1,2, \ldots, n$. But for every $x \in I, F_{i}(x, 0) \geq f(x, 0)=1$. By the condition (2), $c_{i} x=0, i=$ $1,2, \ldots, n$. Hence $x \in \mathbf{r}\left(c_{1}, \ldots, c_{n}\right)$. This shows that $I \subseteq \mathbf{r}\left(c_{1}, \ldots, c_{n}\right)$. On the contrary, if $x \in$ $\mathbf{r}\left(c_{1}, \ldots, c_{n}\right)$, then $F_{i}(x, 0)=F_{i}\left(x, c_{i} x\right)>0$. So $f(x, 0)=\min \left\{F_{1}(x, 0), \ldots, F_{n}(x, 0)\right\}>0$. Hence $x \in I$. This shows that $\mathbf{r}\left(c_{1}, \ldots, c_{n}\right) \subseteq I$. Therefore, $I=\mathbf{r}_{R}\left(c_{1}, \ldots, c_{n}\right)$. So $R$ is a right CF ring.

The following proposition can be looked on as an approach to the CF conjecture.

Proposition 4.2 Let $R$ be a ring. If for every extendable $f \in \operatorname{WFHom}_{R}\left(R_{R}, R_{R}\right)$, there exist $F_{1}, \ldots, F_{n} \in \operatorname{FHom}_{R}\left(R_{R}, R_{R}\right)$ such that $f=F_{1} \wedge F_{2} \wedge \cdots \wedge F_{n}$. Then $R$ is right artinian.

Proof By Theorem 4.1, $R$ is a right $\mathrm{CF}$ ring. It is well known that a right $\mathrm{CF}$ and right noetherian ring is right artinian. So we only need to prove that $R$ is right noetherian.

Assume $R$ is not right noetherian, then there is a strictly ascending chain $I_{1} \subsetneq I_{2} \subsetneq I_{3} \ldots$ of right ideals of $R$. Let $f$ be a fuzzy subset of $R \times R$ constructed by

$$
f(x, y)= \begin{cases}1, & x \in I_{1} \text { and } y=0 \\ \frac{1}{k}, & k \geq 2, x \in I_{k}, x \notin I_{k-1} \text { and } y=0, \\ 0, & \text { others. }\end{cases}
$$

By Example 2.8, $f \in \operatorname{WFHom}_{R}\left(R_{R}, R_{R}\right) . f$ is extendable and not bounded. So there exist some $F_{1}, \ldots, F_{n} \in \mathrm{FHom}_{R}\left(R_{R}, R_{R}\right)$ such that $f=F_{1} \wedge F_{2} \wedge \cdots \wedge F_{n}$. According to the condition (1), there exist $c_{i} \in R$ such that $F_{i}\left(1, c_{i}\right)>0, i=1,2, \ldots$. Then by the condition (4), $F_{i}\left(x, c_{i} x\right) \geq F_{i}\left(1, c_{i}\right)>0$. So $F_{i}(x, y)=0$ or $F_{i}(x, y) \geq F_{i}\left(1, c_{i}\right)$ for all $i=1,2, \ldots, n$. Thus, $f=F_{1} \wedge F_{2} \wedge \cdots \wedge F_{n}$ is bounded. This is a contradiction. So $R$ is right noetherian. Thus, $R$ is right artinian.

Remark 4.3 According to Theorem 3.2, Theorem 4.1 and Proposition 4.2, the CF conjecture is equivalent to saying that every extendable $f \in \mathrm{WFHom}_{R}\left(R_{R}, R_{R}\right)$ of a right CF ring $R$ is bounded.

At last, we obtain a fuzzy characterization of QF rings. 
Theorem 4.4 $R$ is a $Q F$ ring if and only if, for every $f \in \operatorname{WFHom}_{R}\left(R_{R}, R_{R}\right)$, there exist $F_{1}, \ldots, F_{n} \in \operatorname{FHom}_{R}\left(R_{R}, R_{R}\right)$ such that $f=F_{1} \wedge F_{2} \wedge \cdots \wedge F_{n}$.

Proof Suppose $R$ is a QF ring. Then $R$ is right artinian and the right $R$-module $R_{R}$ is injective. By Theorem 3.2, every $f \in \operatorname{WFHom}_{R}\left(R_{R}, R_{R}\right)$ is finite valued. So $f$ is bounded. Since $R_{R}$ is injective, by Theorem 3.1, $f$ is extendable. As QF rings are right CF rings, by Theorem 4.1, there exist $F_{1}, \ldots, F_{n} \in \mathrm{FHom}_{R}\left(R_{R}, R_{R}\right)$ such that $f=F_{1} \wedge F_{2} \wedge \cdots \wedge F_{n}$.

Conversely, if for every $f \in \operatorname{WFHom}_{R}\left(R_{R}, R_{R}\right)$, there exist $F_{1}, \ldots, F_{n} \in \operatorname{FHom}_{R}\left(R_{R}, R_{R}\right)$, such that $f=F_{1} \wedge F_{2} \wedge \cdots \wedge F_{n}$. By Proposition 4.2, $R$ is right artinian. According to Definition 2.4, $f \leq F_{1}$. Then by Theorem 3.1, $R_{R}$ is injective. So $R$ is a QF ring.

Competing interests

The authors declare that they have no competing interests.

Authors' contributions

The three authors contributed equally in writing this article. They read and approved the final manuscript.

\section{Author details}

${ }^{1}$ Department of Mathematics and Physics, Anhui University of Technology, Ma'anshan, 243002, China. ${ }^{2}$ Department of Mathematics, Southeast University, Nanjing, 210096, China

\section{Acknowledgements}

The authors would like to thank the referees for their nice suggestions and comments. It is supported by NSFC (No. 11371089), NSF of Jiangsu Province (No. BK20130599), NSF of Anhui Province (No. 1408085MA04), the Project-sponsored by SRF for ROCS, SEM and Specialized Research Fund for the Doctoral Program of Higher Education (No. 20120092110020).

Received: 22 February 2014 Accepted: 28 April 2014 Published: 13 May 2014

\section{References}

1. Zadeh, LA: Fuzzy sets. Inf. Control 8, 338-353 (1965)

2. Liu, WJ: Fuzzy invariant subgroups and fuzzy ideals. Fuzzy Sets Syst. 8, 133-139 (1982)

3. Golan, JS: Making modules fuzzy. Fuzzy Sets Syst. 32, 91-94 (1989)

4. López-Permouth, SR, Malik, DS: On categories of fuzzy modules. Inf. Sci. 52, 211-220 (1990)

5. López-Permouth, SR: Lifting Morita equivalence to categories of fuzzy modules. Inf. Sci. 64, 191-201 (1992)

6. Malik, DS: Fuzzy ideals of artinian rings. Fuzzy Sets Syst. 37, 111-115 (1990)

7. Malik, DS, Mordeson, JN: Fuzzy homomorphisms of rings. Fuzzy Sets Syst. 46, 139-146 (1992)

8. Mukherjee, TK, Sen, MK: Rings with chain conditions. Fuzzy Sets Syst. 39, 117-123 (1991)

9. Rosenfeld, A: Fuzzy groups. J. Math. Anal. Appl. 35, 512-517 (1971)

10. Nakayama, T: On Frobeniusean algebras, I. Ann. Math. 40,611-633 (1939)

11. Faith, C, Huynh, DV: When self-injective rings are QF: a report on a problem. J. Algebra Appl. 1, 75-105 (2002)

12. Greferath, M, Nechaev, A, Wisbauer, R: Finite quasi-Frobenius modules and linear codes. J. Algebra Appl. 3, 247-272 (2004)

13. Nechaev, A: Linear codes and polylinear recurrences over finite rings and quasi-Frobenius modules. Dokl. Akad. Nauk. 345, 229-254 (1995)

14. Nicholson, WK, Yousif, MF: Quasi-Frobenius Rings. Cambridge University Press, Cambridge (2003)

15. Shen, L, Chen, JL: New characterizations of quasi-Frobenius rings. Commun. Algebra 34, 2157-2165 (2006)

16. Tachikawa, H: Quasi-Frobenius Rings and Generalizations. Springer, New York (1973)

17. Gómez-Pardo, JL, Guil-Asensio, PA: When are all the finitely generated modules embeddable in free modules? In: Lewin, RA (ed.) Rings, Hopf Algebras, and Brauer Groups. Lecture Notes in Pure and Appl. Math., vol. 197, pp. 209-217. Dekker, New York (1998)

18. Li, WX, Chen, JL: When CF rings are artinian. J. Algebra Appl. 12, 1250059 (2013)

19. Anderson, FW, Fuller, KR: Rings and Categories of Modules. Springer, New York (1992) 